1武汉美捷登生物科技有限公司

or 研究发现：抑郁会加速大脑老化影响记忆力

一项新的研究显示, 受抑有症困扰的人随着年龄增长, 更 容易产生记忆力问题。

多达 $25 \%$ 的老年人患有抑郁症, 这反过来又与加速大脑衰 老过程的大脑变化有关。迈阿密大学的最新发现是抑郁症与老年 痴呆症联系的众多研究之一, 但由于二者有很多共同的症状, 因 此难以分辨因果关系。

通常, 患有阿尔兹海默症的病人也患有抑有症, 特别是在疾 病早期阶段, 患者对他们的状况相当清楚, 抑郁症和阿尔兹海默 症都表现为退出人群、失去对活动以及对其他人的兴趣、难以专 注或思考的倾向。虽然有很多研究发现抑郁症和记忆力下降的联 系, 但解释这两者关系的科学才刚刚开始。

美国杨百翰大学的一项研究表明, 抑有症会削弱人们区分相 似事物的能力, 使患者很难进行回忆, 但是大部分人在抑郁症早 期, 可以通过服用药物和谈话治疗来治愈, 而越早治愈, 对大 脑的损害会越小。根据迈阿密大学的研究, 发现抑郁症患者的大 脑明显不同于未患有抑郁症的人, 而抑郁症越严重, 脑容量越小, 发生血管病变的风险便会增加 55\%, 或者脑部和脑部血管发生异 常, 如果血管破裂或阻塞, 就会发生中风。

\section{Go 世卫组织拟五年内全面禁用反式脂肪消除健康隐患}

世界卫生组织 (WHO) 计划将于 2023 年彻底消除工业生产 的反式脂肪。世界卫生组织表示, 目前世界上每年约有 50 万人 因心血管疾病而丧生，推行上述计划将有利于解决这一问题。

世界卫生组织表示, 反式脂肪拥有保存期长的特点, 因此 深受油炸、烤焙以及小吃商的喜爱。但是研究证明, 反式脂肪对 人类健康有害, 将导致消费者患心脏病概率上升 $21 \%$, 死亡概率 上升 $28 \%$ 。

世界卫生组织总干事 Tedros Adhanom Ghebreyesus 发表声 明称：“我们的孩子不应该吃这些含有有害物质的食物。”他表 示, 世界卫生组织将全面贯彻该战略, 积极开发并在全世界推广 更健康的反式脂肪替代物, 并且将推动立法禁止在食物中使用有 害原料, 确保这些有害物质不得进入食物销售链中。

\section{Gor 香港高校研发防贝类过敏疫苗防敏效力至少半年}

据香港《大公报》报道, 不少人对贝壳类海鲜有食物敏感, 有 80 后女患者便曾因呼吸困难等过敏反应, 吃虾蟹蚝后五次送
院抢救。香港中文大学针对贝类过敏研究免疫疗法, 研发疫苗一 针的防敏效力达六个月至一年, 甚至脱敏, 让患者可尽情吃贝类 海鲜。小鼠实验显示, 打三针效力达三周, 研究团队正招揽贝类 过敏者, 预料两年后临床试验。

香港中文大学医学院儿科学系博士后研究员卫懿欣及其团 队, 2011 年展开贝类过敏研究, 发现低致敏性致敏原 MEM49 可减低原肌球蛋白引致的过敏反应。在小鼠体内注射三次 MEM49 疫苗, 其免疫球蛋白 E 降低 76\%, 并诱导体内耐受性细 胞及调节性细胞增长, 从而减少免疫球蛋白 $\mathrm{E}$ 依附于原肌球蛋白。 她解释, 过敏反应源于体内免疫球蛋白 $\mathrm{E}$ 依附于一种叫原肌球 蛋白的致敏原, 疫苗作用是减少两者依附, 甚至完全无依附。

\section{Gor 从大医疗到大健康医改再出发}

如果把我国医疗卫生体制改革当作诊疗对象，全国政协委 员、北京大学党委副书记、医学部党委书记刘玉村给出 3 条诊断： 有限度的财力投入与日益增长的多元化需求间的矛盾、医改成效 的社会整体评价与个体感受间的差异、不同社会群体之间的利益 平衡, “这是一道世界难题”

医改一直在路上。李克强总理在今年的政府工作报告中共 有 27 次提到 “医” 字。3 月 13 日提请全国人民代表大会审议的 国务院机构改革方案中，作出了重要调整——组建国家卫生健康 委员会、组建国家医疗保障局。

\section{Gor 普通体检不能代替防癌筱查}

据了解, 自世界卫生组织明确提出了癌症的早发现、早诊断、 早治疗的 “三早” 策略以来, 癌症的篮查和早诊早治, 已被公认 为癌症防控最有效的途径。

目前, 中国癌症的早诊率低于西方发达国家, 与人们在认识 上的误区有一定关系。临床中，经常遇到患者因为恐惧肿瘤的发 生, 而忽视身体征兆甚至拒绝必要的定期检查, 从而导致对肿 瘤早期诊断和治疗的延误。这也在一定程度上, 造成我国癌症患 者 5 年生存率偏低, 而死亡率相对较高。其实, 通过主动积极 的预防和干预, 了解自己的高危因素、定期进行预防咨询、30 岁 以后每年做个体化防癌体检, 都是提高早诊率的有效途径。

需要特别强调的是, 肿瘤早期不一定会出现明显症状, 常难 以及时察觉, 千万不能以自查代替体检, 而且, 肿瘤篮查与健康 查体的内容也不完全一致, 因此, 普通体检也不能代替防癌篮查。 